\title{
MDO-Based Concept Modelling and the Impact of Fuel Systems on Wing Design
}

\author{
Steven R. H. Dean ${ }^{*}$, John J. Doherty ${ }^{\dagger}$ and Thomas R. Wallace ${ }^{\ddagger}$ \\ Aerospace Consulting, QinetiQ Ltd, Farnborough, Hampshire, GU14 OLX, England
}

\begin{abstract}
During the early stages of aircraft design it is typically the case that potential solutions are down-selected based upon a relatively low level of supporting information. It is also often the case that major airframe characteristics are effectively fixed by the time serious consideration is given to the integration of aircraft systems. As a result, the integration of systems may be significantly more complex and may lead to greater performance penalties than would be the case if these integration requirements were better addressed during early design. The evolution of new capabilities for supporting early aircraft design, based upon Multi-disciplinary Design Optimisation (MDO) toolsets, can potentially enable more complex requirements to be directly addressed in early design. In order to assess the potential opportunities resulting from the use of MDO, consideration has been given to the requirement for improved modelling of fuel systems within early civil aircraft wing design. Details of the MDO approach used are presented. Enhancements to the MDO method, associated with the integration of fuel tanks within the wing subject to uncontained engine rotor failure (UERF) considerations, are described. The results of an initial parametric variation study are presented. The reported work has been carried out as part of the Integrated Wing collaborative programme, which is part funded by the UK Department for Business, Enterprise \& Regulatory Reform (DBERR).
\end{abstract}

\section{Introduction}

$\mathrm{T}$ ypically during the early stages of aircraft design relatively low fidelity concept studies, supported by limited higher fidelity preliminary design activities, are carried out to assess potential solutions. At this stage of design the objective is to down-select a final aircraft concept which is predicted to best meet the overall aircraft project requirements. The definition of the selected aircraft concept is then very significantly refined in the subsequent stages of design resulting in the final manufacturing definition.

It is obviously not ideal to base critical concept design decisions, such as the configuration layout and associated technology and systems choices, together with the prediction of concept performance, upon information which is insufficiently detailed and immature, since this introduces significant risk for the overall aircraft project. More detailed information will be generated using the design toolsets employed in the later stages of design, but frequently these toolsets are manually intensive and hence have not been suitable for use within the short timescales expected during the early stages of design, particularly for application to many possible design alternatives. Where initial design decisions and performance predictions are subsequently found to be inaccurate, it is usually too late to fundamentally rethink the concept selection, so resolving such problems often leads to increased complexity, increased costs and time delays for the aircraft project as a whole.

The aerospace design process is facing even greater challenges for addressing the design of future aircraft. Operational performance drivers are becoming increasingly demanding, potentially leading to the future adoption of more novel aircraft configurations, employing novel technologies and systems within an overall more integrated solution. In particular stringent environmental targets for civil aircraft, such as the ACARE 2020 Vision $^{1}$ for $50 \%$ reduction in $\mathrm{CO} 2$ and $80 \%$ reduction in NOX, mean that novel aircraft configurations such as blended wing body concepts, employing novel technologies, such as flow control and extensive use of composites, must be considered.

\footnotetext{
${ }^{*}$ Senior Engineer, Member AIAA.

${ }^{\dagger}$ QinetiQ Fellow, Visiting Professor Surrey University, Senior Member AIAA.

${ }^{*}$ Engineer, Member AIAA.
} 
The addition of these novel factors into the existing non-ideal design process means alternative approaches for supporting early design are required, which can improve confidence in performance predictions and design decisions and can identify the best combination of aircraft configuration, technologies and systems.

In recent years new design capabilities, based upon the use of a Multi-disciplinary Design Optimisation (MDO) approach, have emerged. Although an MDO based approach can be used at many different stages of aircraft design, the focus here is upon usage during the early stages of design. In comparison to the conventional aircraft design process, MDO offers the potential to assess a wider range of design freedoms simultaneously, to facilitate improved modelling fidelity within a relatively rapid automated process and to incorporate more detailed design constraints to give better integrated solutions. The work described here specifically focuses on the use of MDO for enabling better integration of technologies and systems within early aircraft design. In particular it addresses the impact on the selection of an aircraft concept as a result of improved modelling of fuel systems during design.

\section{Integrated Wing Project}

The Integrated Wing Aerospace Technology Validation Programme ${ }^{2}$ (IWATVP) brings together industry and researchers within a UK national project funded jointly by the UK Department for Business, Enterprise \& Regulatory Reform (DBERR) and the participating industrial partners. The structure of the Integrated Wing programme covers all of the primary technologies relevant to civil aircraft wing design, including aerodynamics, loads, structures, fuel systems, landing gear, power distribution and health monitoring, as shown in figure 1. The overall aim of the project is to validate technologies which can lead to a step change in performance for future aircraft, in order to address challenging future operational performance requirements best characterised by the ACARE 2020 Vision.

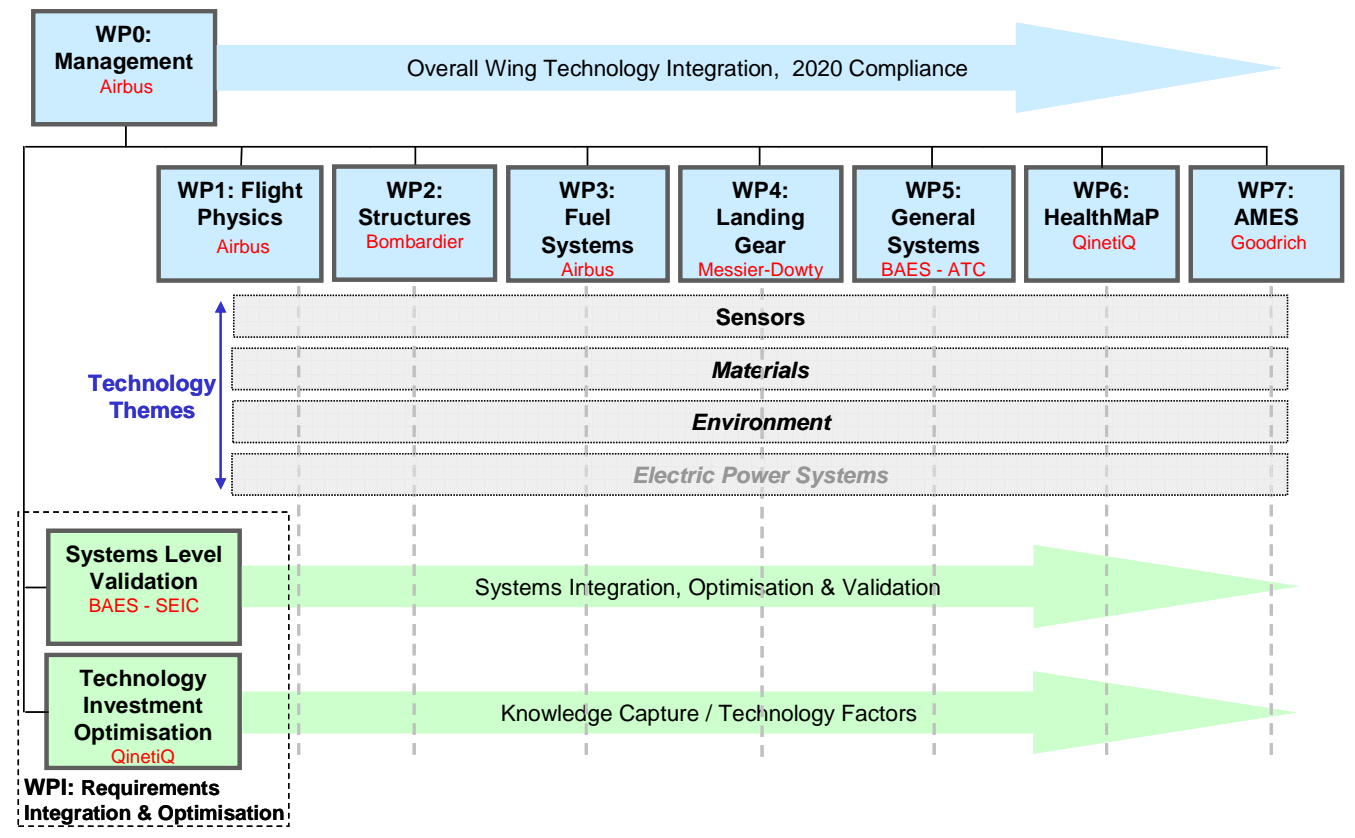

Figure 1. The Integrated Wing Programme Structure

As part of the Integrated Wing programme QinetiQ leads the Work Package focused on Requirements Integration and Optimisation (WPI), as shown in Figure 1. A key objective for QinetiQ within WPI is to research and demonstrate the potential for alternative analysis and design approaches to support decision making in the early stages of design. Capabilities for process automation and optimisation, operating at different levels of modelling fidelity, are reasonably well established at QinetiQ and have been used for a number of years for military air vehicle design studies. Within WPI QinetiQ is assessing the potential of these alternative approaches for both conventional and novel civil aircraft concepts, encompassing associated conventional and novel technologies and system choices.

Two approaches for supporting design decisions are being investigated within WPI. The first addresses the selection of technologies and systems in the context of broad design space exploration, which is described in reference 3. The second approach, which will be reported in detail within this paper, enables a more detailed 
investigation of the impact of technology and system choices for specific aircraft concept types using a Multidisciplinary Design Optimisation approach. Initially the objective for this work is to generate understanding of how an aircraft conceptual design, and its associated performance, will change if a technology or system is better integrated from the outset. This enables the true value of a technology or system to be identified.

The work reported here covers fuel systems specific activities being carried out as part of the wider Integrated Wing programme.

\section{Multi-disciplinary Design Optimisation Process}

Within the Integrated Wing project QinetiQ work is building upon its existing Multi-Disciplinary Concept Assessment and Design (MDCAD) capability ${ }^{4-7}$. The original development of the baseline MDCAD capability was driven by the need to broaden the applicability and improve the accuracy of existing aircraft conceptual design methods to cover novel aircraft configurations, whilst maintaining a sufficiently fast design process. As part of the Integrated Wing project this baseline capability is being further extended to cover the integration of detailed technologies and systems, through improved physical modelling and additional design freedoms and constraints.

The baseline MDCAD capability incorporates a wide range of discipline based performance prediction models together with whole aircraft mission modelling. It uses a 'fit for purpose' mix of computational physics based performance prediction models, such as Computational Fluid Dynamics (CFD) and Finite Element Analysis (FEA), together with low-fidelity, semi-empirical models where appropriate. MDCAD facilitates earlier use of physics based performance prediction models, combined with more detailed definition of aircraft concepts, to support design studies for novel concepts, technologies and systems, which were previously beyond the range of applicability of previous low-fidelity approaches. The low-fidelity and high-fidelity performance prediction models that have been incorporated within MDCAD, together with optimisation tools, are drawn from proven single disciplinary capabilities spread across many different domains within QinetiQ.

MDCAD represents a reconfigurable design environment, which is extensively automated to enable numerical optimisation driven design to be completed, in order to incorporate the required level of design maturity with reduced man effort. The environment consists of two main parts:

- Software framework for allowing new performance models and optimisation tools to be linked to give an efficient automated analysis and design process. This framework must be sufficiently flexible to enable the implementation of complex multi-disciplinary design problem formulations and associated design knowledge. The framework must also address significant data exchange requirements and the need to ensure efficient computational implementation.

- A central aircraft geometry representation which can be used concurrently by the many models integrated within the framework. This geometry representation uses a rules-based, parametric CAD model generator, to enable both design variations to be generated automatically and also allows existing design knowledge to be incorporated as part of the overall design problem formulation. It also acts as a geometry analysis model delivering 'measure' type performance attributes such as separation distance, area, volume, centre of gravity and inertia.

The framework and the geometry representation must support the use of multiple levels of fidelity in order to enable the most efficient 'fit for purpose' modelling approach. In addition data exchange and performance accounting must allow the effect of design changes to be synchronised across the various performance models, such that the impact of design changes can be measured at the overall aircraft mission level.

A bespoke framework utilising Python has been developed and is used to automate the process. The rules-based, parametric CAD model generator is based upon the CATIA V5 commercial software product from Dassault Systèmes, enabling the automated generation of full external aircraft surfaces, structural layouts, local surface features (e.g. blending), deployable devices and internal packaging and systems. Computational physics analysis and optimisation tools are interfaced with this central CAD model within the software framework, to enable rapid analysis and optimisation. The exchange of information between the disciplines is standardized, for example the aerodynamics/structures exchange of loads and aero-elastic displacements. The baseline MDCAD framework used within the Integrated Wing project, which as highlighted previously is focussed upon civil aircraft wing technologies, is shown schematically in Figure 2. The wing and belly fairing components, including their location, are modelled parametrically, whilst the remaining aircraft components, such as the fuselage, empennage and engine, are primarily fixed. 


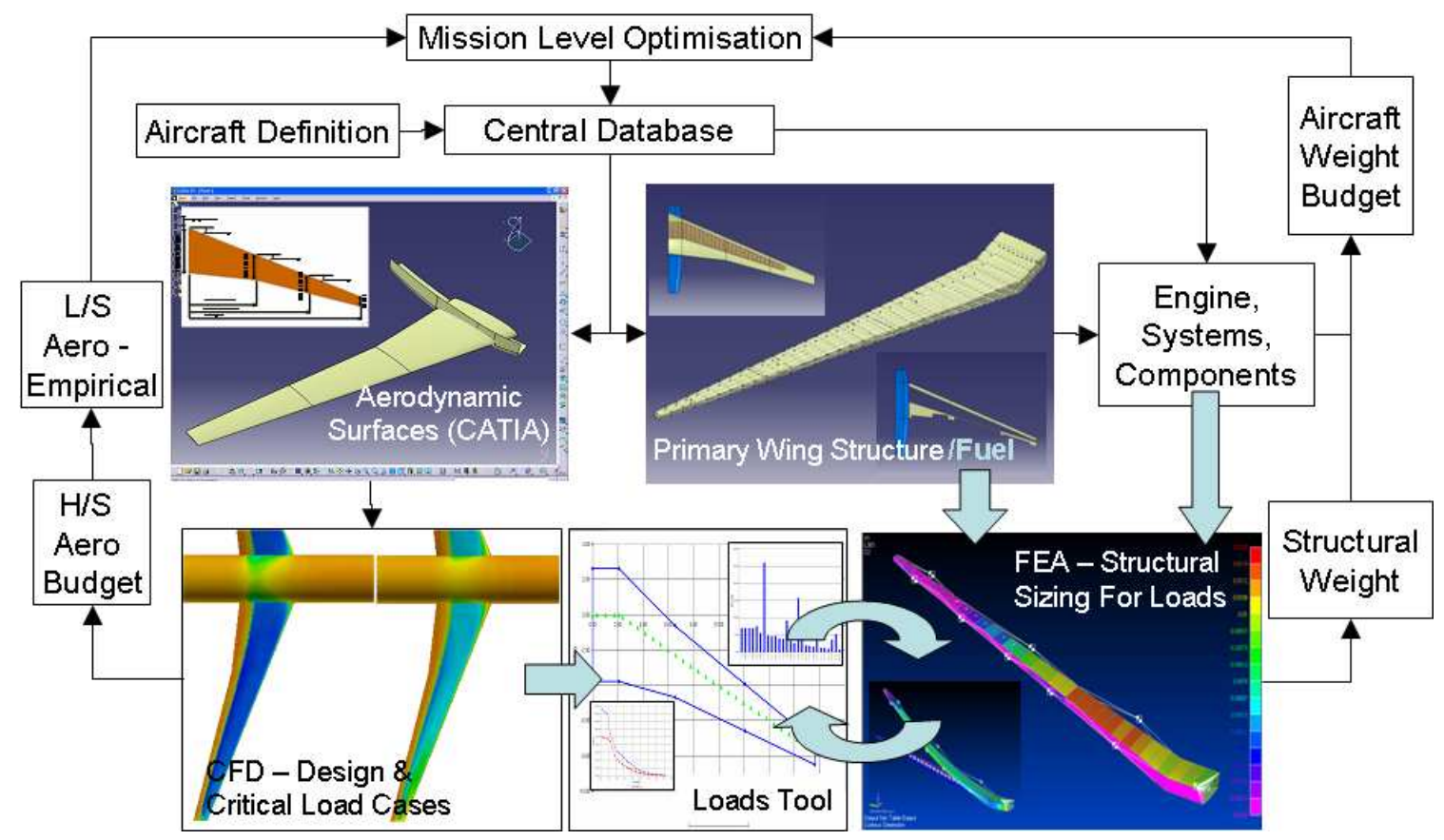

Figure 2. The Baseline MDCAD Framework

The wing component is defined in terms of typical configuration level parameters, controlling the wing planform, its location and the position and size of moveable devices. Additionally, more detailed parameters define the external wing shape (e.g. local camber, thickness, twist) and the belly fairing shape and location. For overall aircraft aerodynamic trim the horizontal tail-plane setting angle is also available as a parameter. Detailed parameters also control the layout of the primary wing structure (e.g. local spar, rib and stringer locations and sizing) and the useable fuel volume. These parameters are used to drive the CATIA V5 rules-based CAD geometry generator, which creates the external wing surface, belly fairing, moveable devices (including tail-plane) and the internal wing structural geometry. CATIA V5 is also used to directly create an FEA compatible representation of the wing structure for later use with MSC NASTRAN. The maximum capacity available for fuel within each wing bay is also calculated within CATIA for subsequent use.

The external CAD surfaces are analyzed using CFD to generate aerodynamic performance information together with aerodynamic loading information. The primary wing structural components (spar, ribs, skin) are analyzed and their thicknesses optimized using MSC NASTRAN SOL200, to provide the basis for subsequent structural weight predictions. During the structural analysis of the wing the aerodynamic loads are applied, together with loads associated with fuel carriage and other possible wing components e.g. engine installation, high-lift systems, aileron and landing gear.

The aerodynamic performance predictions from CFD, the weight predictions from FEA and other aircraft components, together with details of the chosen engine e.g. specific fuel consumption, are fed back into the overall optimisation problem formulation. A mission analysis module is used to calculate the performance of the aircraft from which various optimisation objective functions can be derived e.g. total fuel burn, maximum range, minimum weight etc. Additional constraints, such as ensuring the lift to weight balance at cruise and overall aircraft stability margins and trim, are also included within the process.

Each of the component parts of the overall process are set up as automated processes e.g. CAD generation, CFD grid generation, FEA model generation. The data exchange between these components can also be automated e.g. CAD input to CFD grid generation, transfer of aerodynamic loads into FEA. Hence the overall concept optimisation process can be fully automated and can be run without the requirement for user intervention.

The importance of a framework for linking the overall MDO process together is clear from Figure 3. There is frequently a requirement to establish an optimisation process across a network of machines. For example the CATIA V5 software is used on Windows platforms at QinetiQ, whilst the CFD and FEA software is usually run on a Linux multi-processor cluster. QinetiQ also uses Python to establish an overall optimisation process across a network of machines. 
Within the MDCAD process the use of optimisation, and the associated higher-fidelity analysis methods, to directly support concept analysis and design means that the conventional boundaries between conceptual design and preliminary design have been removed, and the two phases have to a large extent been merged. By using physics based analysis methods rather than simpler historical based correlations, the MDCAD approach provides generality to enable novel concepts to be assessed and designed, and improves the accuracy of the performance levels assumed during the conceptual design phase. This approach also results in the output of concepts which are more compatible with later stages of design helping to reduce overall design cycle.

Past applications of MDCAD have typically demonstrated the importance of optimising configuration level parameters (eg wing planform) and detailed parameters (eg aerodynamic shaping, structural sizing) simultaneously, subject to mission constraints (eg range, low-speed performance, stability) and packaging constraints (eg fuel volume). This has involved simultaneous geometric, aerodynamic, loads and structural design. Overall improvements in aircraft performance are the result of a balance being derived between geometric considerations (eg fuel volume), aerodynamic design (changes to the wing planform and local shaping), associated modified aerodynamic loading and structural design (sizing of structural components driven by modified loading).

\section{Aircraft Fuel Systems}

The integration of fuel systems within an aircraft imposes many complex constraints upon the overall design. At the early stages of design an initial layout of the fuel systems must be incorporated which satisfies a number of constraints that influence aircraft performance, refuelling times and safety. In particular these factors have an impact on the shape of the wing, the wing structural layout and sizing and the position of the centre of gravity of the aircraft for calculation of the Basic Operating Sequence (BOS). It is hence desirable to more fully model these constraints within the MDCAD process in order to better represent the drivers for aircraft concept design.

The key drivers that impact the layout of the fuel system are:

- Uncontained Engine Rotor Failure

- Fuel system packaging

- Fuel management to provide wing aeroelastic bending relief and aerodynamic trim

- Protection of the fuel system against lightening strike

\section{A. Uncontained Engine Rotor Failure}

Aircraft powered by turbine engines are subject to the possibility of a rotor failure where fragments from the fan, compressor or turbine sections are ejected at high velocity from the engine. When designing the fuel systems layout, these fragments are assumed to contain enough energy and be of such a size that they can not be contained within the engine casing. It is therefore assumed that the fragments can pass through any aircraft component encountered along the trajectories taken by the fragments with the potential for catastrophic results. This is referred to as an uncontained engine rotor failure (UERF).

To minimise the impact of such a failure aircraft safety regulating authorities, such as the European Aviation Safety Agency (EASA) and the Federal Aviation Authority (FAA), have drawn up a set of certification requirements which aircraft manufacturers must adhere to. For large aircraft, EASA certification specifications ${ }^{8}$ state that "Design precautions must be taken to minimise the hazards to the aeroplane in the event of an engine rotor failure". One of the main hazards following UERF is the potential loss of stored fuel and as a result this impacts on the design of the aircraft fuel tank layout. The amount of fuel lost following UERF can be minimised by correctly positioning the tank boundaries such that there is always enough fuel to ensure that the aircraft can reach a diversionary airfield and land safely.

The EASA regulations, within reference 9, contain a section specifically addressing the impact of UERF on aircraft design (AMC 20-128A "Design Considerations for Minimizing Hazards Caused by Uncontained Turbine Engine and Auxiliary Power Unit Rotor Failure"). These regulations provide manufacturers with a description of the models and methods that are necessary to analyse UERF events. In particular three different fragment types are defined, which are representative of those that might be encountered during an UERF event:

- Single One-Third Disc fragment

- Intermediate Fragment

- Small Fragments

These fragment types, together with the anticipated trajectory spread angle associated with each fragment type, is shown in Figure 3. 


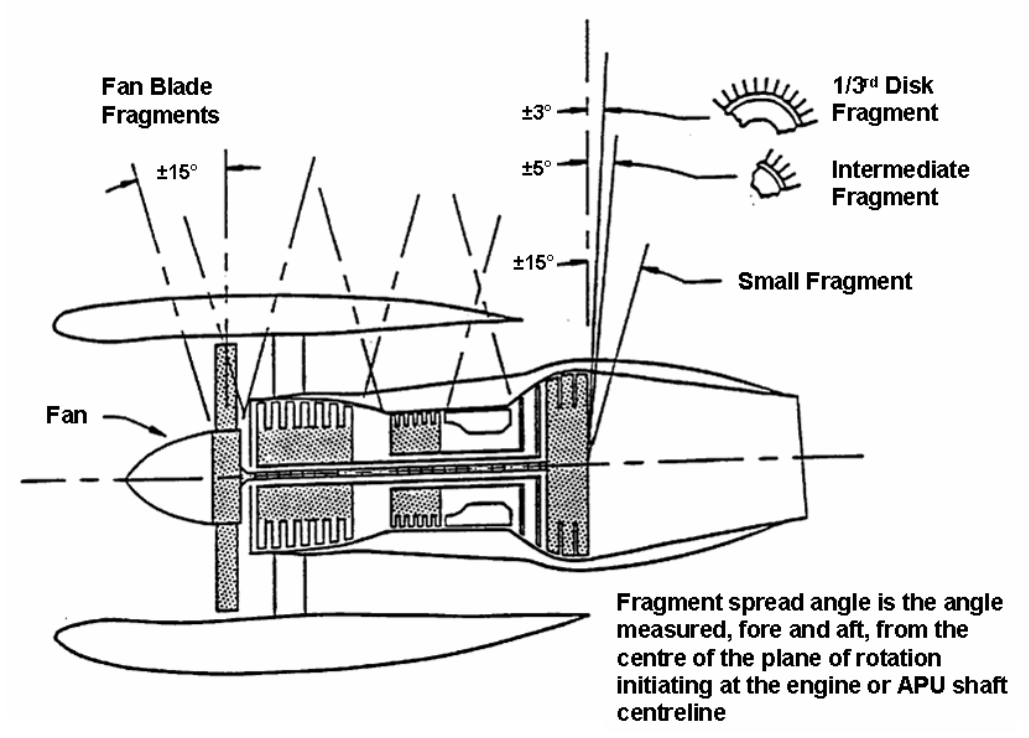

Figure 3. Fragment Definitions (taken from reference 9).

Reference 9 also defines a series of models which a fuel system designer can use to assess the trajectory of fragments ejected. In particular, section (9)(c) in reference 9, entitled "Alternative Engine Model", states that analysis of an UERF event can be assessed based on "a single one-third disk fragment with a spread angle of $\pm 5^{\circ}$ ". Also section 4.1 (a) of Appendix 1 of reference 9 states that "the fragments ejected posses infinite energy making them capable of severing lines, wiring, cables and unprotected structure".

The above guidelines, when mapped onto an aircraft representation will create zones that are susceptible to impact from an ejected rotor fragment. A fuel system designer can then position fuel tank boundaries so as to minimise the loss of fuel in the event of UERF fragments rupturing the wing skin within these zones. These tank boundaries will often be located at wing ribs, so the integration of UERF constraints may lead to the need to position wing ribs in order to define the extent of fuel tank boundaries.

\section{B. Fuel System Packaging}

It is necessary during the design of the wing to ensure that there is sufficient internal volume available, both for carriage of the required amount of fuel and also to incorporate physical components associated with the fuel system, such as pumps, valves and piping associated with distribution, refuelling and venting. Hence there will be a trade off to be made between the aerodynamic and structural performance versus the size and location of these components.

\section{Fuel Management}

During all flight conditions for which the wings are generating positive lift there will be a vertical, aerodynamic load acting on the wing structure, which is tending to bend the wing structure upwards, resulting in a maximum displacement at the wing tip. The wing structure must hence be sized to bear this bending load, with the weight of the resulting wing structure being highly dependent upon the maximum size of this aerodynamic bending load. The weight of the fuel stored within the wing fuel tanks can be used to counteract this bending load, which will result in a lower, net load acting on the wing structure (wing bending relief), with an associated reduction in the designed wing structural weight. Modern aircraft actively manage the distribution of fuel around the various fuel tanks in order to optimise the wing bending relief over the duration of a flight.

As the fuel is used during flight aircraft with swept wings can experience changes in the location of the centre of gravity during a mission, as the mass and centre of gravity of the fuel in the wing changes. These changes in the aircraft centre of gravity will impact upon the stability of the aircraft. The position of the aerodynamic centre of lift would need to be modified, in line with changes to the centre of gravity, to maintain overall aircraft stability. Such changes to the aerodynamic balance are possible using the tail and wing devices, but this will lead to undesirable aerodynamic trim drag penalties as the aerodynamic devices deviate from their ideal design settings. By positioning a trim fuel tank at the rear of the aircraft, fuel management can be used to control the centre of gravity of the aircraft, without the need to alter the aerodynamic balance, hence avoiding a penalty in aerodynamic drag. 


\section{Lightening Strike Protection}

Aircraft may be exposed to lightening strikes during normal operating conditions. Reference 8 includes certification requirements, which must be taken into account during design, to ensure that the aircraft fuel system is protected against the possibility of fuel vapour ignition, as a result of either direct lightening strike, swept lightening strokes, or corona and streaming at fuel vent outlets. Whilst this requirement will primarily relate to the need for electrical bonding of the wing structure and components, it may also have an impact upon the positioning and packaging of fuel system components.

\section{Fuel Systems Modelling Within MDCAD}

Within the Integrated Wing programme a number of enhancements have been made to the baseline MDCAD capability, in order to better represent the requirements for fuel systems integration within early aircraft concept design. To date these enhancements address:

- Generation of detailed fuel volumes and locations

- Fuel management modelling

- Initial modelling of UERF constraints and the impact upon fuel tank layout

- Generic approach for including individual fuel system packaging constraints

\section{A. Generation of detailed fuel volumes and locations}

Initial enhancements involved the generation of more detailed fuel volume information using the central geometry modelling within MDCAD. This development was primarily achieved within CATIA V5 building upon the existing parametric CAD definition of the wing. In particular a scripted approach was developed for automatically deriving the maximum volume and centre of gravity of each wing fuel bay from the parametric CAD model generated within MDCAD. Figure 4 shows an example parametric wing CAD model as generated within

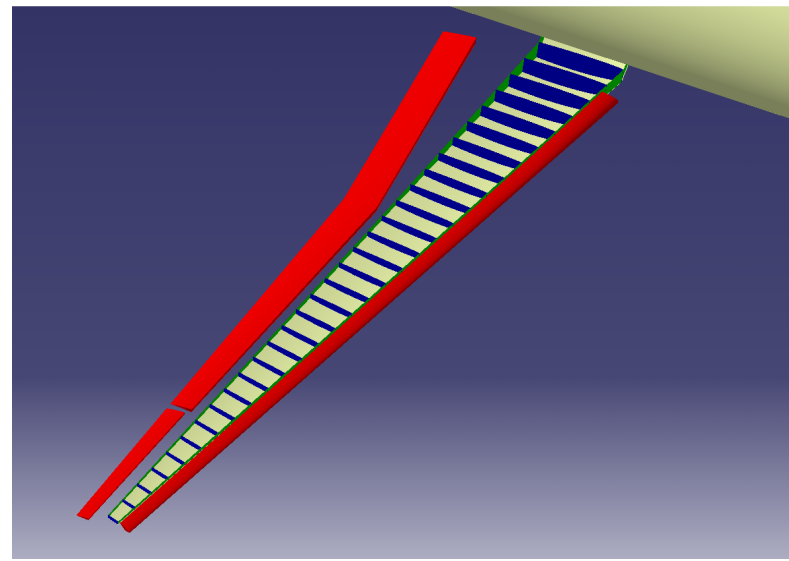

Figure 4. Parametric wing CAD model showing individual wing fuel bays.
MDCAD. The wing fuel bays correspond to the volume bounded by the front and rear spars and each set of neighbouring ribs. It should be noted that this volume is an idealised maximum, since it would be reduced if the physical thickness of structural components were modelled within the CAD definition. Additionally, in practice as the fuel in a bay is nearly exhausted some of the available volume will not be useable because local shaping may lead to regions where fuel 'pools' remote from a local fuel pump inlet. Engineering judgement is used to make reasonable assumptions and estimates about the maximum useable fuel volume within each wing bay.

The generation of more detailed fuel volumes within MDCAD provides a benefit in terms of improved accuracy for the prediction of the overall maximum available fuel capacity, which is used within the mission modelling.

\section{B. Fuel Management}

The individual wing bay volumes described above can also be used in a variety of ways to improve fuel systems modelling within MDCAD. Multiple bays can be combined together to define an outline fuel tank arrangement. As will be seen in the following UERF section this choice of which wing bays to assign to which fuel tanks can be driven automatically as part of the overall MDCAD design process.

The definition of the overall fuel tank layout, together with the definition of the volumes and centres of gravity of each wing bay within each fuel tank, can be used as part of a fuel management strategy model within MDCAD. This can allow the volume of fuel in each aircraft fuel tank to be varied in order to represent different fuel load operating situations, such as for take-off, beginning and end of cruise etc. In turn, this allows variation of the fuel loading applied to the FEA structural sizing during design, in order to represent different wing bending relief conditions and the impact on structural sizing and hence structural weight. Currently a simple fuel management strategy is used within MDCAD, corresponding to a small number of different fuel load and associated flight load 
conditions. Within the FEA structural sizing process within MDCAD, these fuel loads are used to partly offset the corresponding aerodynamic loading. By using the additional fuel tank layout information and the associated volumes and centres of gravity, this modelling of critical load conditions can be further improved with an associated improvement in FEA structural sizing process.

The modelling of the fuel contained within individual fuel tanks, corresponding to different operating situations, also provides the basis for predicting the associated shift in the fuel centre of gravity. If a trim fuel tank is available then a fuel management strategy model can utilise this trim tank to potentially alleviate the shift in the aircraft centre of gravity. This in turn will avoid the need to aerodynamically trim the aircraft using the tail-plane, which will hence avoid the associated aerodynamic trim drag penalty. This use of a trim tank has not yet been implemented within the fuel management strategy model within MDCAD, although the key data required to implement this modelling is now available as part of the process.

\section{Initial modelling of UERF constraints and the impact upon fuel tank layout}

In order to implement initial modelling of UERF constraints within MDCAD, it was first necessary to implement an automatic means for identifying regions of the aircraft geometry which would be affected by a UERF event. This was again mainly implemented by extending the central parametric geometry modelling within MDCAD. In particular, the location of a wing mounted engine is already available as part of the central geometry model. For UERF modelling it was also necessary to define the location of the engine shafts and the longitudinal extent of the rotating engine components from which fragments may eminate. As described earlier, reference 9 allows a UERF event to be modelled as a single one-third disk fragment with a spread angle of $\pm 5^{\circ}$. The combination of the engine shaft location, the shaft extent and the spread angle of $\pm 5^{\circ}$ allowed a fragment trajectory volume to be defined automatically for each engine, as part of the parametric CAD model within CATIA V5. An example of these fragment trajectory regions is shown in figure 5 .
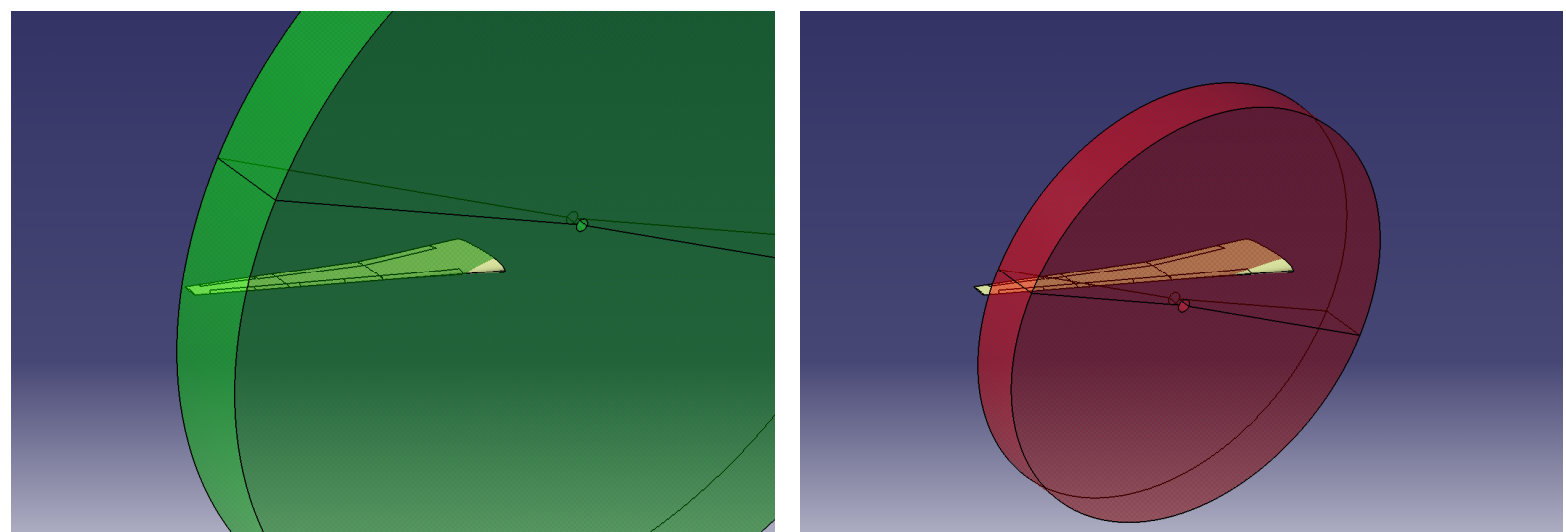

Figure 5. UERF fragment trajectory regions modelled within MDCAD. Left hand figure shows region for port engine. Right hand figure shows region for starboard engine.

The region of the wing which will be impacted by a UERF event is dictated by both the engine mounted on the local wing and by the engine mounted on the opposite wing. This can be seen more clearly in figure 6 which shows a planform view of the wing, together with the regions of the wing planform which are affected by the UERF fragment trajectories.

In order to identify the specific fuel bay regions which would be affected by a UERF event, a further parametric process was implemented within the central CAD modelling within MDCAD. In particular, the CAD representation of the individual wing fuel bays are intersected by each of the fragment trajectory regions. Each wing fuel bay is then automatically identified as either being within, or partly within, a region (and hence affected) or fully outside (and hence not affected). In order to show this more clearly, figure 7 shows two example wings generated within MDCAD, which differ by $2^{\circ}$ sweep. Figure 7 also shows the individual fuel bay regions, where the red colouring corresponds to those bays that are affected by UERF fragments from both engines, the green colouring shows the bays solely affected by the region eminating from the engine on the opposite wing, the yellow and blue colouring shows the bays that are unaffected. By comparing the green coloured fuel bays for the wing in figure $7 \mathrm{a}$ with that in figure $7 \mathrm{~b}$, it can be seen that increasing wing sweep by $2^{\circ}$ results in the most outboard green wing fuel bay switching to yellow, indicating that it is no longer affected by the UERF constraint for the increased wing sweep case. 

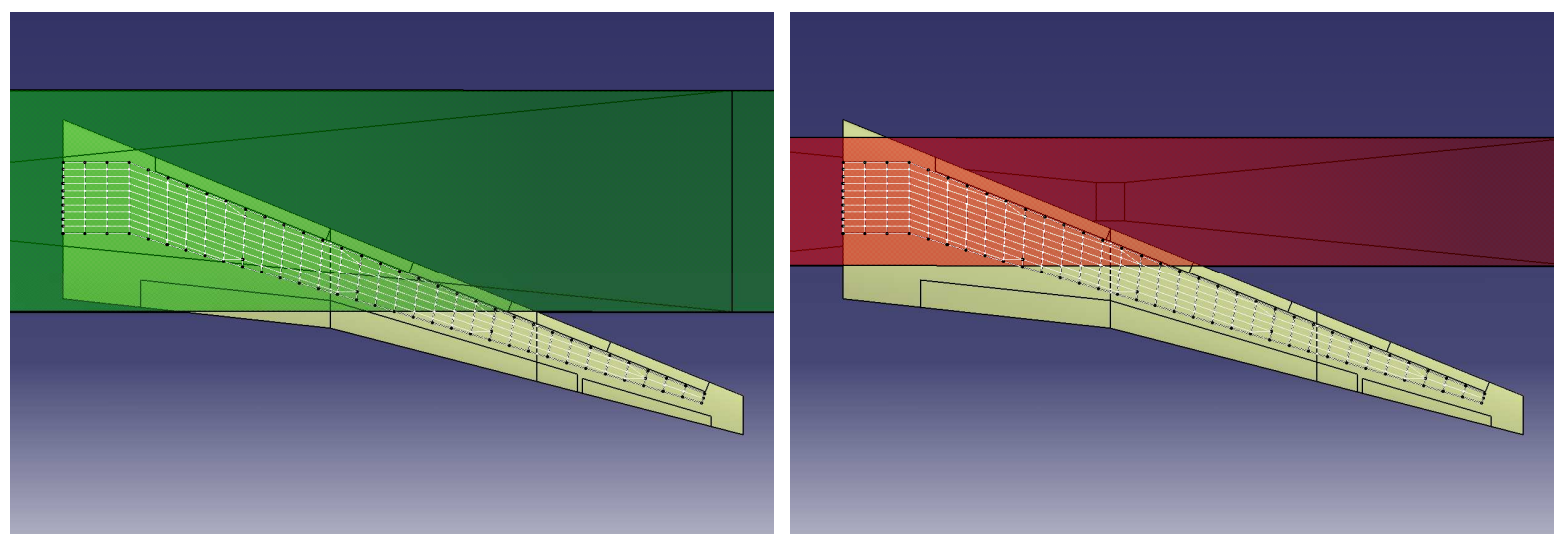

Figure 6. Planform view of UERF fragment trajectory regions modelled within MDCAD. Left hand figure shows region for port engine. Right hand figure shows region for starboard engine.

Based on the example shown in figure 7 it can be seen that sufficient information is now available to help define a sensible aircraft fuel tank layout and in particular to define the bounds of individual aircraft fuel tanks. For example if the divide between seperate fuel tanks is located at start of the unaffected wing fuel bays (start of yellow coloured bays in figure 7), then the yellow region will define the volume of fuel which will still be useable after a UERF event. In practice, a fuel tank arrangement also requires a surge tank, which acts as an 'overflow' tank to cater for expansion of the fuel in normal operation - fuel entering this surge tank will be subsequently pumped back into the aircraft fuel tanks once space becomes available. This surge tank is therefore not available as useable fuel volume. An example surge tank definition is shown as the blue region within figure 7 . By applying rules which dictate the allocation of wing fuel bays to individual fuel tanks, an outline fuel tank arrangement can be generated automatically as part of the MDCAD process. This fuel tank layout can then be used as part of the fuel management modelling described earlier to model the affects of fuel load relief for the structural design and similarly to assess the change in fuel centre of gravity for trim.
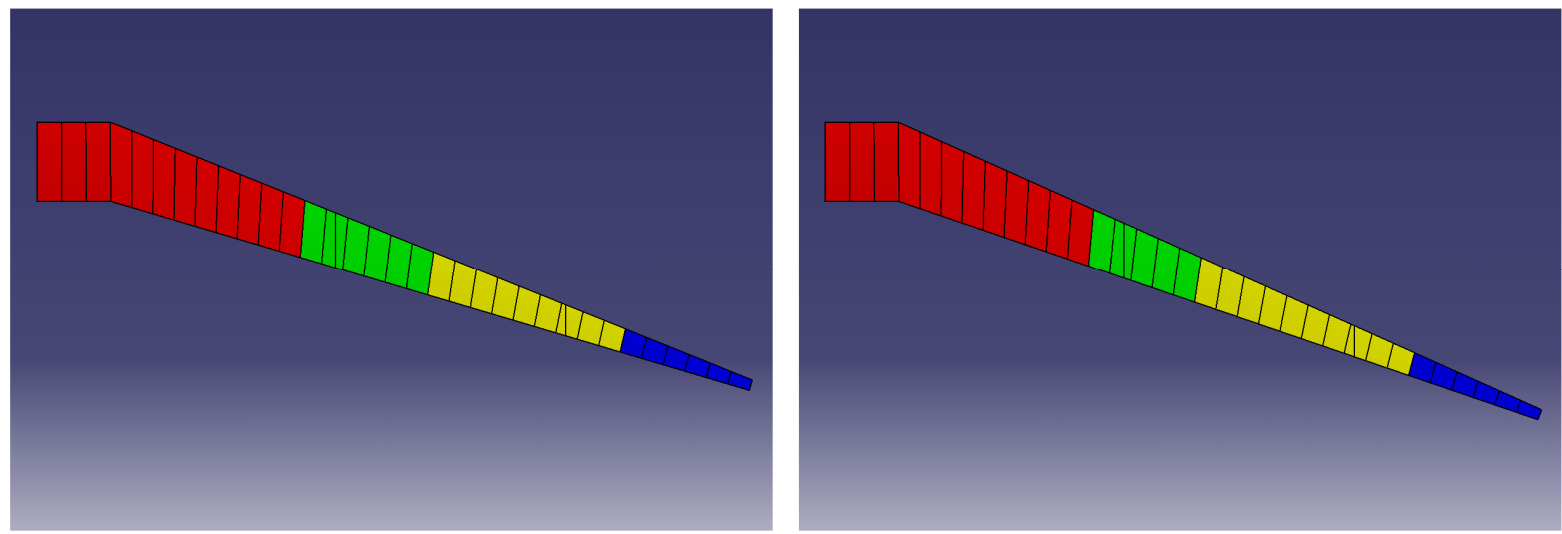

Figure 7. Wing fuel bays affected by UERF fragments: (a) baseline wing planform (b) wing sweep increased by $2^{\circ}$. Red colouring corresponds to impact of fragments from both engines, green colouring corresponds to impact from the engine on the opposite wing, yellow shows the bays that are unaffected, blue shows the bays assigned to the fuel surge tank. 


\section{Generic Approach For Including Individual Fuel System Packaging Constraints}

The final enhancement, for improving the modelling of fuel

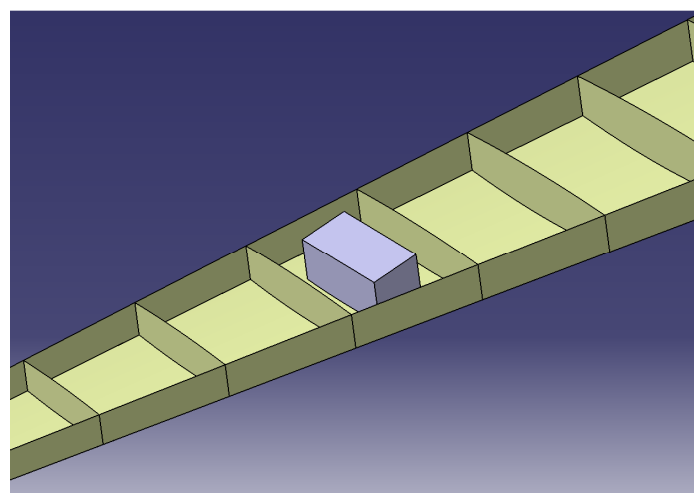

Figure 8. Generic fuel system component modelled as a packaging constraint systems within MDCAD, provides a means for dealing with packaging constraints associated with individual fuel system components eg pumps, valves and piping associated with distribution, refuelling and venting. To provide a generic means for dealing with these fuel system geometric constraints, individual components of the fuel system can be defined by a simple length, breadth and height packaging size, together with a location within the wing. Each component is then modelled as a simple box inside the central MDCAD parametric CAD model. Figure 8 shows a simple packaging representation for a generic fuel system component. CATIA V5 can be used to evaluate the minimum separation between the internal component and the wing geometry, or to identify a maximum overlap, which is then used with an optimisation constraint. By this means the wing geometry will be constrained to fit around each internal component during the design process.

Specific enhancements for protection against lightening strike have not to date been modelled within MDCAD. However where these requirements lead to location or local packaging considerations, then it is possible to cater for these using the same generic approach for packaging constraints.

\section{Parameter Variation Study}

In order to initially assess the application of the new fuel systems modelling within the MDCAD process, a design parameter variation study has been completed. Various key design parameters controlling the wing geometry, the structural layout and the fuel tank extent were assigned appropriate increments relative to a baseline aircraft definition. The MDCAD process was run for all of the required parametric variations in order to predict the effect on the overall aircraft performance metrics and to test the robustness of the new fuel systems parametric modelling. The associated results for a selection of design parameters associated with wing planform changes (leading edge sweep, wing root chord, wing semi-span), structural layout (rib pitch) and the ratio of the span available for fuel tanks (fuel span), are presented in figure 9. A significant amount of data is generated for each iteration of the process, so only a limited selection of data is plotted.

In figure 9 , the variable delta indicates the size of the parametric variation relative to the parameter starting value, MTOW refers to the aircraft maximum take-off weight, $\mathrm{L} / \mathrm{D}$ is the aerodynamic lift/drag ratio at mid-cruise, $\mathrm{L} / \mathrm{W}$ is the ratio of the aircraft aerodynamic lift to the aircraft weight at mid-cruise and trim refers to the overall aircraft aerodynamic trim at mid-cruise. It should be noted that these parametric variations do not represent a viable design change, since only one variable is changed at a time and the aircraft is not redesigned to give a self-consistent solution for each parametric change. For example, it can be seen from figure 9 that individual parametric variations lead to changes in the aircraft range, the lift/weight ratio and the aircraft trim. In practice, during a full design optimisation each of these performance characteristics would be modelled as constraints, such that they achieve target values as part of the design process. In particular, the range is likely to be a fixed requirement rather than an objective, the aerodynamic lift should be equal to the aircraft weight at cruise (ie L/W should have a value of unity) and the aircraft aerodynamic trim at mid-cruise should be equal to zero (ie L/D ratio corresponds to trimmed midcruise condition).

In the context of the work described here, the key outputs of interest are the fuel volume (the available fuel capacity) and the post UERF fuel volume (the volume of fuel remaining in the tanks not affected by the UERF trajectory spread angles. In particular it can be seen in figure 9 that increasing the leading edge sweep has little affect on the available fuel volume (wing thickness is same as the baseline wing so internal volume is unchanged), while it has a significant affect of the volume of fuel available following a UERF event. This is in keeping with the increase in the post UERF fuel volume following an a small increase in sweep described earlier and shown in figure 7. Likewise an increase in the wing root chord length provides an increase in the available fuel volume for the inner wing region, but does not provide additional fuel volume on the outboard wing and hence does not affect the post UERF fuel volume. 


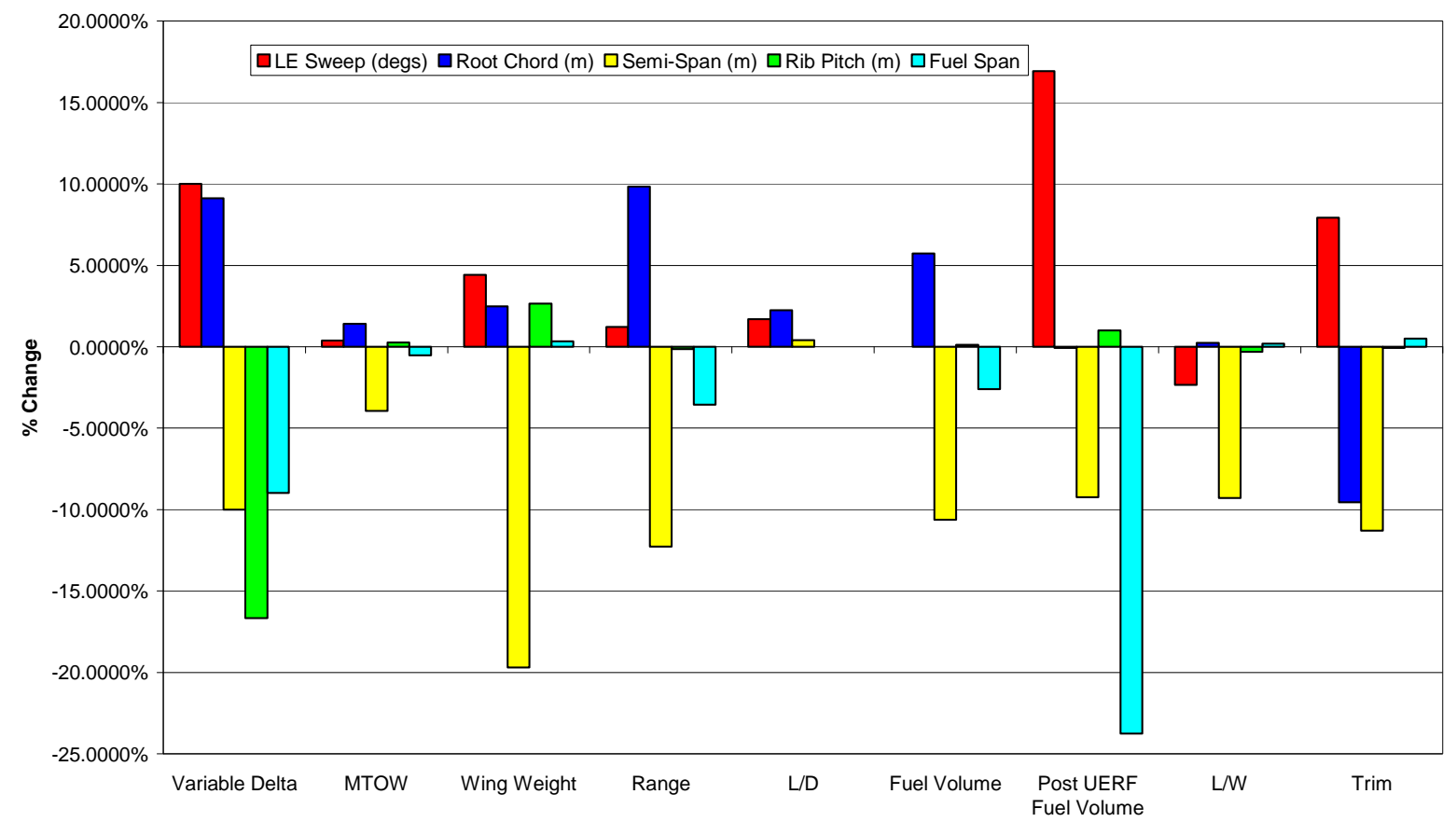

Figure 9. Design parameter variation study.

The parametric variation study demonstrates the ability of the process to automatically assess the impact of integrating additional fuel systems modelling and also demonstrates that the drivers for fuel systems integration may potentially be quite different to the parameters that influence the total fuel volume.

\section{Conclusions}

This paper has described activities completed to date by QinetiQ as part of the UK Integrated Wing programme. A number of developments have been made to a civil aircraft concept design process. The design process uses an MDO based approach, which enables high-fidelity modelling and greater concept detail to be used in the early stages of design. The specific objective for the work described here has been to improve the representation of fuel systems integration requirements within the overall wing design process. In particular, the approach aims to better address the requirements for integrating fuel systems in parallel with, rather than subsequent to, the aerodynamic and structural design of the wing. The improved design process has been initially demonstrated by means of a parametric variation study, which provides promising insights into the potential benefits of the new modelling approach for helping to identify those design parameters which may be most critical for fuel systems integration. This new MDO process will be applied in full optimisation mode in the near future in order to demonstrate the impact that better modelling of fuel systems requirements has on early civil aircraft wing design.

\section{Acknowledgements}

QinetiQ work within the Integrated Wing programme is funded jointly by the UK Department for Business, Enterprise and Regulatory Reform (DBERR) and QinetiQ.

\section{References}

\footnotetext{
${ }^{1}$ ACARE, Strategic Research Agenda 1 \& 2, URL: www.acare4europe.org [cited 23 December 2008]

${ }^{2}$ Integrated Wing Aerospace Technology Validation Programme, URL: www.integrated-wing.org.uk [cited 23 December 2008]

${ }^{3}$ Ellsmore, P. D., Restrick, K. E., "Application of RETIVO to Civil Aircraft", $7^{\text {th }}$ AIAA ATIO Conference, No. AIAA-20077808, Belfast, North Ireland, September 2007.
} 
${ }^{4}$ Hackett, K. C., Rees, P. H., Chu, J. K., “Aerodynamic Design Optimisation Applied to Civil Transports with Underwing Mounted Engines", in proceedings of $21^{\text {st }}$ ICAS Conference, Melbourne, Australia, September 1998.

${ }^{5}$ Fenwick, S. V., Harris, J. ap C., "The application of Pareto frontier methods in the multidisciplinary wing design of a generic modern military delta aircraft", in proceedings of Symposium of the NATO RTO Applied Vehicle Technology Panel (AVT), Ottawa, Canada, October 1999.

${ }^{6}$ Doherty, J. J., Dean, S. R. H., "The Role of Design Optimisation within the Overall Vehicle Design Process", in proceedings of 6th ASMO UK / ISSMO conference on Engineering Design Optimization, Oxford, England, July 2006.

${ }^{7}$ Doherty, J. J., Dean, S. R. H., Ellsmore, P. D., Eldridge, A., “A Multi-Fidelity Approach for Supporting Early Aircraft Design Decisions", in proceedings of the 15th ISPE International Conference on Concurrent Engineering (CE2008), Belfast, Northern Ireland, August 2008.

${ }^{8}$ European Aviation Safety Agency, "Certification Specifications for Large Aeroplanes CS-25", Subpart E Powerplant, Section 903, Engines (d)(1), Amendment 5 dated 5 September 2008.

9 European Aviation Safety Agency, "Executive Director Decision On General Acceptable Means Of Compliance For Airworthiness Of Products, Parts And Appliances AMC-20”, ED Decision 2003/12/Rm, 5 November 2003. 